\title{
GENERALIZATIONS OF SOME POLYNOMIAL INEQUALITIES FOR THE FAMILY OF $B$-OPERATORS
}

\author{
Gulshan Singh, W. M. Shah And A. Liman
}

Abstract. Let $P_{n}$ be the class of polynomials of degree at most $n$. In 1969, Rahman [Functions of exponential type, Trans. Amer. Math. Soc., 135(1969), 295-309] introduced a class $B_{n}$ of operators $B$ that map $P_{n}$ into itself and proved that

$$
\max _{|z|=1}|B[P(R z)]| \leqslant\left|B\left[E_{n}(R z)\right]\right| \max _{|z|=1}|P(z)|, \quad R \geqslant 1,
$$

for every $B \in B_{n}$, where $E_{n}(z):=z^{n}$.

In this paper, we prove some generalizations and refinements of this result, which in particular yields some known polynomial inequalities as special cases.

Mathematics subject classification (2010): 30A10, 30C10, 30C15.

Keywords and phrases: Polynomials, $B$-operator, inequalities.

\section{Introduction and statement of results}

Let $P_{n}$ be the class of polynomials $P(z):=\sum_{j=0}^{n} a_{j} z^{j}$ of degree at most $n$ and $P^{\prime}(z)$ its derivative, then it is known that

$$
\max _{|z|=1}\left|P^{\prime}(z)\right| \leqslant n \max _{|z|=1}|P(z)|
$$

and

$$
\max _{|z|=R>1}|P(z)| \leqslant R^{n} \max _{|z|=1}|P(z)| .
$$

Inequality (1), which is an immediate consequence of Bernstein's inequality (for reference see [6]) on the derivative of a trigonometric polynomial is best possible with equality holding for the polynomial $P(z)=\lambda z^{n}$, where $\lambda$ is a complex number. Inequality (2) is a simple deduction from the maximum modulus principle (see [13, p. 346], [9, p. 158], problem 269).

For the class of polynomials $P \in P_{n}$ having all their zeros in $|z| \leqslant 1$, we have

$$
\min _{|z|=1}\left|P^{\prime}(z)\right| \geqslant n \min _{|z|=1}|P(z)|
$$

and

$$
\min _{|z|=R>1}|P(z)| \geqslant R^{n} \min _{|z|=1}|P(z)| .
$$

Inequalities (3) and (4) are due to Aziz and Dawood [3]. Both the results are sharp and equality holds for a polynomial having all its zeros at the origin. 
If we restrict ourselves to a class of polynomials having all their zeros in $|z| \geqslant 1$, inequalities (1) and (2) can be sharpened. In fact, if $P(z) \neq 0$ in $|z|<1$, then

$$
\max _{|z|=1}\left|P^{\prime}(z)\right| \leqslant \frac{n}{2} \max _{|z|=1}|P(z)|
$$

and

$$
\max _{|z|=R>1}|P(z)| \leqslant\left(\frac{R^{n}+1}{2}\right) \max _{|z|=1}|P(z)| .
$$

Inequality (5) was conjectured by Erdôs and later verified by Lax [7], where as Ankeny and Rivlin [1] used (5) to prove (6). Inequalities (5) and (6) were further improved in [3] and under the same hypothesis, it was shown that

$$
\max _{|z|=1}\left|P^{\prime}(z)\right| \leqslant \frac{n}{2}\left\{\max _{|z|=1}|P(z)|-\min _{|z|=1}|P(z)|\right\}
$$

and

$$
\max _{|z|=R>1}|P(z)| \leqslant\left(\frac{R^{n}+1}{2}\right) \max _{|z|=1}|P(z)|-\left(\frac{R^{n}-1}{2}\right) \min _{|z|=1}|P(z)| .
$$

Equality in (5), (6), (7) and (8) holds for polynomials of the form $P(z)=\alpha z^{n}+\beta$, where $|\alpha|=|\beta|$.

Aziz [2], Aziz and Shah [4] and Shah [14] extended such well known inequalities to the polar derivative of a polynomial $P(z)$ with respect to a point $\alpha$ defined by

$$
D_{\alpha} P(z):=n P(z)+(\alpha-z) P^{\prime}(z)
$$

and obtained several sharp inequalities. Like polar derivative there are many other operators which are just as interesting (for reference see [11,12]). It is an interesting problem, as pointed out by Professor Q. I. Rahman to characterize all such operators. As a part of this characterization Rahman [10] (see also Rahman and Schmeisser [12, page 538-551]) introduced a class $B_{n}$ of operators $B$ that map $P \in P_{n}$ into itself. That is, the operator $B$ carries $P \in P_{n}$ into

$$
B[P](z):=\lambda_{0} P(z)+\lambda_{1}\left(\frac{n z}{2}\right) \frac{P^{\prime}(z)}{1 !}+\lambda_{2}\left(\frac{n z}{2}\right)^{2} \frac{P^{\prime \prime}(z)}{2 !}
$$

where $\lambda_{0}, \lambda_{1}, \lambda_{2}$ are real or complex numbers, such that all the zeros of

$$
u(z):=\lambda_{0}+c(n, 1) \lambda_{1} z+c(n, 2) \lambda_{2} z^{2}, \quad c(n, r)=\frac{n !}{r !(n-r) !}
$$

lie in the half plane

$$
|z| \leqslant\left|z-\frac{n}{2}\right|
$$

and observed:

THEOREM A. If $P \in P_{n}$, then

$$
\max _{|z|=1}|B[P(R z)]| \leqslant\left|B\left[E_{n}(R z)\right]\right| \max _{|z|=1}|P(z)|, \quad R \geqslant 1 .
$$

As an improvement Shah and Liman [15] proved: 
Theorem B. If $P \in P_{n}, P(z) \neq 0$ for $|z|<1$, then

$$
|B[P(R z)]| \leqslant \frac{1}{2}\left\{\left|B\left[E_{n}(R z)\right]\right|+\left|\lambda_{0}\right|\right\} \max _{|z|=1}|P(z)|,
$$

for every $B \in B_{n}$, where $E_{n}(z):=z^{n}$.

Theorems A and B provide compact generalizations of inequalities (1), (2) and (3), (4) respectively and these inequalities follow when we substitute for $B[P](z)$ and then use $\lambda_{0}, \lambda_{1}$ and $\lambda_{2}$ suitably.

In this paper, we prove some more general results concerning the operator $B \in B_{n}$ preserving inequalities between polynomials, which in turn yields compact generalizations of some well known polynomial inequalities. We first prove:

THEOREM 1. Let $F(z)$ be a polynomial of degree $n$ having all zeros in $|z| \leqslant k$, where $k \geqslant 0$ and $f(z)$ be a polynomial of degree not exceeding that of $F(z)$. If $|f(z)| \leqslant$ $|F(z)|$ for $|z|=k$, then for all complex numbers $\alpha, \beta$ with $|\alpha| \leqslant 1,|\beta| \leqslant 1, R>r \geqslant k$ and $|z| \geqslant 1$, we have

$$
|B[f(R z)]+\psi(R, r, \alpha, \beta, k) B[f(r z)]| \leqslant|B[F(R z)]+\psi(R, r, \alpha, \beta, k) B[F(r z)]|,
$$

where

$$
\psi(R, r, \alpha, \beta, k):=\beta\left\{\left(\frac{R+k}{r+k}\right)^{n}-|\alpha|\right\}-\alpha .
$$

A variety of interesting results can be deduced from Theorem 1 as special cases. For example, by taking $k=1$, we immediately have the following:

COROLlaRY 1. Let $F(z)$ be a polynomial of degree $n$ having all its zeros in $|z| \leqslant 1$ and $f(z)$ be a polynomial of degree not exceeding that of $F(z)$. If

$$
|f(z)| \leqslant|F(z)| \text { for }|z|=1,
$$

then for any real or complex numbers $\alpha, \beta$ with $|\alpha| \leqslant 1,|\beta| \leqslant 1, R>r \geqslant 1$ and $|z| \geqslant 1$, we have

$$
|B[f(R z)]+\phi(R, r, \alpha, \beta) B[f(r z)]| \leqslant|B[F(R z)]+\phi(R, r, \alpha, \beta) B[F(r z)]|,
$$

where

$$
\phi(R, r, \alpha, \beta):=\beta\left\{\left(\frac{R+1}{r+1}\right)^{n}-|\alpha|\right\}-\alpha .
$$

The following result immediately follows from Theorem 1 by taking $f(z)=P(z)$ and $F(z)=M z^{n}$, where $M=\max _{|z|=1}|P(z)|$.

COROLlARY 2. If $P(z)$ is a polynomial of degree $n$, then for all real or complex numbers $\alpha, \beta$ with $|\alpha| \leqslant 1,|\beta| \leqslant 1, R>r \geqslant k \geqslant 0$, we have

$$
\begin{aligned}
& |B[P(R z)]+\psi(R, r, \alpha, \beta, k) B[P(r z)]| \\
\leqslant & \frac{1}{k^{n}}\left|B\left[E_{n}(R z)\right]+\psi(R, r, \alpha, \beta, k) B\left[E_{n}(r z)\right]\right| \max _{|z|=k}|P(z)|,
\end{aligned}
$$


where $\psi$ and $E_{n}$ are defined above.

In particular for $k=1$, we have the following interesting result:

COROLlaRY 3. Let $P(z)$ be a polynomial of degree $n$, then for all real or complex numbers $\alpha, \beta$ with $|\alpha| \leqslant 1,|\beta| \leqslant 1, R>r \geqslant 1$,

$$
\begin{aligned}
& |B[P(R z)]+\phi(R, r, \alpha, \beta) B[P(r z)]| \\
\leqslant & \left|B\left[E_{n}(R z)\right]+\phi(R, r, \alpha, \beta) B\left[E_{n}(r z)\right]\right| \max _{|z|=1}|P(z)| \text { for }|z| \geqslant 1,
\end{aligned}
$$

where $\phi$ and $E_{n}$ are defined above.

For $\alpha=0$ in Corollary 3, we get the following:

COROLlaRY 4. Let $P(z)$ be a polynomial of degree $n$, then for every real or complex number $\beta$ with $|\beta| \leqslant 1, R>r \geqslant 1$,

$$
\begin{aligned}
& \left|B[P(R z)]+\beta\left(\frac{R+1}{r+1}\right)^{n} B[P(r z)]\right| \\
\leqslant & \left|B\left[E_{n}(R z)\right]+\beta\left(\frac{R+1}{r+1}\right)^{n} B\left[E_{n}(r z)\right]\right| \max _{|z|=1}|P(z)| \text { for }|z| \geqslant 1 .
\end{aligned}
$$

REMARK 1 . For $\beta=0$, Corollary 4 reduces to inequality (12). Next if we chose $\lambda_{1}=\lambda_{2}=0$ and $\beta=0$ in (18) and note that all the zeros of $u(z)$ defined by (10) lie in the region (11), we obtain for every real or complex number $\alpha$ with $|\alpha| \leqslant 1, R>r \geqslant 1$,

$$
|P(R z)-\alpha P(r z)| \leqslant\left|R^{n}-\alpha r^{n}\right|\left|z^{n}\right| \max _{|z|=1}|P(z)| \text { for }|z| \geqslant 1 .
$$

Inequality (20) includes inequality (2) as a special case when $\alpha=0$. Further, if we divide both sides of the inequality (20) by $R-r$ with $\alpha=1$ and making $R \rightarrow r$, we get

$$
\left|P^{\prime}(r z)\right| \leqslant n r^{n-1}|z|^{n-1} \max _{|z|=1}|P(z)| \text { for }|z| \geqslant 1
$$

which in particular yields inequality (1).

THEOREM 2. If $P(z)$ is a polynomial of degree $n$, having no zeros in the disk $|z|<k$, where $k \geqslant 0$, then for all real or complex numbers $\alpha, \beta$ with $|\alpha| \leqslant 1,|\beta| \leqslant 1$, $R>r \geqslant k$ and $|z| \geqslant 1$, we get

$$
|B[P(R z)]+\psi(R, r, \alpha, \beta, k) B[P(r z)]| \leqslant|B[Q(R z)]+\psi(R, r, \alpha, \beta, k) B[Q(r z)]|,
$$

where $Q(z):=\left(\frac{z}{k}\right)^{n} \overline{P\left(\frac{k^{2}}{\bar{z}}\right)}$ and $\psi(R, r, \alpha, \beta, k)$ is defined by (15). 
THEOREM 3. If $P(z)$ is a polynomial of degree $n$, having all its zeros in the disk $|z| \leqslant k$, where $k \geqslant 0$, then for all real or complex numbers $\alpha, \beta$ with $|\alpha| \leqslant 1,|\beta| \leqslant 1$, $R>r \geqslant k$, we have

$$
\begin{aligned}
& |B[P(R z)]+\psi(R, r, \alpha, \beta, k) B[P(r z)]| \\
\geqslant & \frac{1}{k^{n}}\left|B\left[E_{n}(R z)\right]+\psi(R, r, \alpha, \beta, k) B\left[E_{n}(r z)\right]\right| \min _{|z|=k}|P(z)|,
\end{aligned}
$$

where $\psi$ and $E_{n}$ are defined above.

THEOREM 4. Let $P(z)$ be a polynomial of degree $n$, then for all real or complex numbers $\alpha, \beta$ with $|\alpha| \leqslant 1,|\beta| \leqslant 1, R>r \geqslant k, k \leqslant 1$ and $|z|=1$, we have

$$
\begin{aligned}
& |B[P(R z)]+\psi(R, r, \alpha, \beta, k) B[P(r z)]|+|B[Q(R z)]+\psi(R, r, \alpha, \beta, k) B[Q(r z)]| \\
\leqslant & \left\{\left|\lambda_{0}\right||1+\psi(R, r, \alpha, \beta, k)|+\frac{1}{k^{n}}\left|B\left[E_{n}(R z)\right]+\psi(R, r, \alpha, \beta, k) B\left[E_{n}(r z)\right]\right|\right\} \max _{|z|=1}|P(z)|,
\end{aligned}
$$

where $Q(z):=\left(\frac{z}{k}\right)^{n} \overline{P\left(\frac{k^{2}}{\bar{z}}\right)}$ and $E_{n}(z):=z^{n}$.

THEOREM 5. Let $P(z)$ be a polynomial of degree $n$ having all its zeros in $|z| \geqslant k$, $k \leqslant 1$, then for all real or complex numbers $\alpha, \beta$ with $|\alpha| \leqslant 1,|\beta| \leqslant 1, R>r \geqslant k$ and $|z|=1$, we have

$$
\begin{aligned}
& |B[P(R z)]+\psi(R, r, \alpha, \beta, k) B[P(r z)]| \\
\leqslant & \frac{1}{2}\left\{\left|\lambda_{0}\right||1+\psi(R, r, \alpha, \beta, k)|+\frac{1}{k^{n}}\left|B\left[E_{n}(R z)\right]+\psi(R, r, \alpha, \beta, k) B\left[E_{n}(r z)\right]\right|\right\} \max _{|z|=1}|P(z)|,
\end{aligned}
$$

where $\psi$ and $E_{n}$ are defined above.

THEOREM 6. Let $P(z)$ be a polynomial of degree $n$ having no zeros in the disk $|z|<k, k \leqslant 1$, then for all real or complex numbers $\alpha, \beta$ with $|\alpha| \leqslant 1,|\beta| \leqslant 1$, $R>r \geqslant k$ and $|z|=1$, we have

$$
\begin{aligned}
& |B[P(R z)]+\psi(R, r, \alpha, \beta, k) B[P(r z)]| \\
\leqslant & \frac{1}{2}\left\{\left[\frac{1}{k^{n}}\left|B\left[E_{n}(R z)\right]+\psi(R, r, \alpha, \beta, k) B\left[E_{n}(r z)\right]\right|+\left|\lambda_{0}\right||1+\psi(R, r, \alpha, \beta, k)|\right] \max _{|z|=1}|P(z)|\right. \\
& \left.+\left[\frac{1}{k^{n}}\left|B\left[E_{n}(R z)\right]+\psi(R, r, \alpha, \beta, k) B\left[E_{n}(r z)\right]\right|-\left|\lambda_{0}\right||1+\psi(R, r, \alpha, \beta, k)|\right] \min _{|z|=k}|P(z)|\right\},
\end{aligned}
$$

where $\psi$ and $E_{n}$ are defined above.

For $\alpha=0$ in Theorem 6 , we have the following: 
COROllary 5. Let $P(z)$ be a polynomial of degree $n$ having no zeros in the disk $|z|<k, k \leqslant 1$, then for every real or complex number $\beta$ with $|\beta| \leqslant 1, R>r \geqslant k$ and $|z|=1$,

$$
\begin{aligned}
& \left|B[P(R z)]+\beta\left(\frac{R+k}{r+k}\right)^{n} B[P(r z)]\right| \\
\leqslant & \frac{1}{2}\left\{\left[\frac{1}{k^{n}}\left|B\left[E_{n}(R z)\right]+\beta\left(\frac{R+k}{r+k}\right)^{n} B\left[E_{n}(r z)\right]\right|+\left|\lambda_{0}\right|\left|1+\beta\left(\frac{R+k}{r+k}\right)^{n}\right|\right] \max _{|z|=1}|P(z)|\right. \\
& \left.+\left[\frac{1}{k^{n}}\left|B\left[E_{n}(R z)\right]+\beta\left(\frac{R+k}{r+k}\right)^{n} B\left[E_{n}(r z)\right]\right|-\left|\lambda_{0}\right|\left|1+\beta\left(\frac{R+k}{r+k}\right)^{n}\right|\right] \min _{|z|=k}|P(z)|\right\} .
\end{aligned}
$$

If we take $\beta=0$ in Theorem 6 , we get

COROLlaRY 6. Let $P(z)$ be a polynomial of degree $n$ having no zeros in the disk $|z|<k, k \leqslant 1$, then for every real or complex number $\alpha$ with $|\alpha| \leqslant 1, R>r \geqslant k$ and $|z|=1$,

$$
\begin{aligned}
& |B[P(R z)]-\alpha B[P(r z)]| \\
\leqslant & \frac{1}{2}\left\{\left[\frac{1}{k^{n}}\left|B\left[E_{n}(R z)\right]-\alpha B\left[E_{n}(r z)\right]\right|+\left|\lambda_{0}\right||1-\alpha|\right] \max _{|z|=1}|P(z)|\right. \\
& \left.+\left[\frac{1}{k^{n}}\left|B\left[E_{n}(R z)\right]-\alpha B\left[E_{n}(r z)\right]\right|-\left|\lambda_{0}\right||1-\alpha|\right] \min _{|z|=k}|P(z)|\right\} .
\end{aligned}
$$

Also, the following result immediately follows from Theorem 6, if we take $k=1$.

COROLlARY 7. Let $P(z)$ be a polynomial of degree $n$ having no zeros in the disk $|z|<1$, then for all real or complex numbers $\alpha, \beta$ with $|\alpha| \leqslant 1,|\beta| \leqslant 1, R>r \geqslant 1$ and $|z|=1$,

$$
\begin{aligned}
& |B[P(R z)]+\phi(R, r, \alpha, \beta) B[P(r z)]| \\
\leqslant & \frac{1}{2}\left\{\left[\left|B\left[E_{n}(R z)\right]+\phi(R, r, \alpha, \beta) B\left[E_{n}(r z)\right]\right|+\left|\lambda_{0}\right||1+\phi(R, r, \alpha, \beta)|\right] \max _{|z|=1}|P(z)|\right. \\
& \left.+\left[\left|B\left[E_{n}(R z)\right]+\phi(R, r, \alpha, \beta) B\left[E_{n}(r z)\right]\right|-\left|\lambda_{0}\right||1+\phi(R, r, \alpha, \beta)|\right] \min _{|z|=1}|P(z)|\right\},
\end{aligned}
$$

where $\phi$ and $E_{n}$ are defined above.

If we take $k=1$ and $\beta=0$ in Theorem 6, we get the following:

COROLlaRY 8. Let $P(z)$ be a polynomial of degree $n$ having no zeros in the disk $|z|<1$, then for all real or complex numbers $\alpha, \beta$ with $|\alpha| \leqslant 1,|\beta| \leqslant 1, R>r \geqslant 1$ and $|z|=1$,

$$
\begin{aligned}
& |B[P(R z)]-\alpha B[P(r z)]| \\
\leqslant & \frac{1}{2}\left\{\left[\left|B\left[E_{n}(R z)\right]-\alpha B\left[E_{n}(r z)\right]\right|+\left|\lambda_{0}\right||1-\alpha|\right] \max _{|z|=1}|P(z)|\right. \\
& \left.+\left[\left|B\left[E_{n}(R z)\right]-\alpha B\left[E_{n}(r z)\right]\right|-\left|\lambda_{0}\right||1-\alpha|\right] \min _{|z|=1}|P(z)|\right\} .
\end{aligned}
$$




\section{Lemmas}

For the proofs of these theorems we require the following lemmas. The first lemma follows from Corollary 18.3 of [8, p. 65].

LEMMA 1. If all the zeros of a polynomial $P(z)$ of degree $n$ lie in a circle $|z| \leqslant k$, where $k \geqslant 0$, then all the zeros of the polynomial $B[P](z)$ also lie in the circle $|z| \leqslant k$ where $k \geqslant 0$.

LEMMA 2. If $P(z)$ is a polynomial of degree $n$, having all zeros in the closed disk $|z| \leqslant k$, where $k \geqslant 0$, then for every $R \geqslant r$ and $r R \geqslant k^{2}$,

$$
|P(R z)| \geqslant\left(\frac{R+k}{r+k}\right)^{n}|P(r z)|, \quad|z|=1 .
$$

The above Lemma is due to Aziz and Zargar [5].

\section{Proofs of the theorems}

Proof of Theorem 1. Since $|f(z)| \leqslant|F(z)|$ for $|z|=k$, therefore any zero of $F(z)$ that lies on $|z|=k$, is also zero of $f(z)$. For $\lambda$ with $|\lambda|<1$, it follows by Rouche's theorem, that the polynomial $H(z)=F(z)+\lambda f(z)$ has all its zeros in $|z| \leqslant k$. On applying Lemma 2 to $H(z)$, we have

$$
H(R z) \geqslant\left(\frac{R+k}{r+k}\right)^{n}|H(r z)|>|H(r z)|, \quad R>r \geqslant k, \quad|z|=1
$$

Therefore, for any $\alpha$ with $|\alpha| \leqslant 1$, we have

$$
|H(R z)-\alpha H(r z)| \geqslant|H(R z)|-|\alpha||H(r z)| \geqslant\left\{\left(\frac{R+k}{r+k}\right)^{n}-|\alpha|\right\}|H(r z)|,|z|=1 .
$$

Since $H(R z)$ has all its zeros in $|z| \leqslant \frac{k}{R}<1$. Therefore, for every real or complex number $\alpha$ with $|\alpha|<1$, it follows from inequality (23) by direct application of Rouche's theorem that the polynomial $H(R z)-\alpha H(r z)$ has all its zeros in $|z|<1$. Again from inequality (24) by the direct application of Rouche's theorem, it follows that for all real or complex number $\beta$ with $|\beta|<1$ and $R>r \geqslant k$, that all the zeros of the polynomial $H(R z)-\alpha H(r z)+\beta\left\{\left(\frac{R+k}{r+k}\right)^{n}-|\alpha|\right\} H(r z)$ lie in $|z|<1$. Applying Lemma 1 and using the linearity of $B$, it follows that all the zeros of the polynomial

$$
T(z):=B[H(R z)]-\alpha B[H(r z)]+\beta\left\{\left(\frac{R+k}{r+k}\right)^{n}-|\alpha|\right\} B[H(r z)]
$$

lie in $|z|<1$ for every real or complex number $\alpha$ with $|\alpha| \leqslant 1$ and $R>r \geqslant k$. Re- 
placing $H(z)$ by $F(z)+\lambda f(z)$, we conclude that all the zeros of the polynomial

$$
\begin{aligned}
T(z):= & B[F(R z)]+\lambda B[f(R z)]-\alpha(B[F(r z)]+\lambda B[f(r z)]) \\
& +\beta\left\{\left(\frac{R+k}{r+k}\right)^{n}-|\alpha|\right\}(B[F(r z)]+\lambda B[f(r z)]) \\
= & B[F(R z)]-\alpha B[F(r z)]+\beta\left\{\left(\frac{R+k}{r+k}\right)^{n}-|\alpha|\right\} B[F(r z)] \\
& +\lambda\left(B[f(R z)]-\alpha B[f(r z)]+\beta\left\{\left(\frac{R+k}{r+k}\right)^{n}-|\alpha|\right\} B[f(r z)]\right)
\end{aligned}
$$

lie in $|z|<1$ for all real or complex numbers $\alpha, \beta$ with $|\alpha| \leqslant 1,|\beta| \leqslant 1, R>r \geqslant k$ and $|z|<1$.

This implies

$$
|B[f(R z)]+\psi(R, r, \alpha, \beta, k) B[f(r z)]| \leqslant|B[F(R z)]+\psi(R, r, \alpha, \beta, k) B[F(r z)]|,
$$

where $\psi(R, r, \alpha, \beta, k):=\beta\left\{\left(\frac{R+k}{r+k}\right)^{n}-|\alpha|\right\}-\alpha,|z| \geqslant 1$ and $R>r \geqslant k$.

If the inequality (25) is not true, then there exist a point $z=\omega$ with $|\omega| \geqslant 1$ such that

$$
|B[f(R z)]+\psi(R, r, \alpha, \beta, k) B[f(r z)]|>|B[F(R z)]+\psi(R, r, \alpha, \beta, k) B[F(r z)]| .
$$

Taking

$$
\lambda=-\frac{B[F(R z)]+\psi(R, r, \alpha, \beta, k) B[F(r z)]}{B[f(R z)]+\psi(R, r, \alpha, \beta, k) B[f(r z)]},
$$

so that $|\lambda|<1$ and with this choice of $\lambda$, we have $T(\omega)=0$ for $|\omega| \geqslant 1$. This is clearly a contradiction to the fact that all the zeros of $T(z)$ lie in $|z|<1$. Thus for all real or complex numbers $\alpha, \beta$ with $|\alpha| \leqslant 1,|\beta| \leqslant 1$ and $R>r \geqslant k$, we get (14).

Proof of Theorem 2. Let $Q(z):=\left(\frac{z}{k}\right)^{n} \overline{P\left(\frac{k^{2}}{\bar{z}}\right)}$. Since all the zeros of a polynomial $P(z)$ of degree $n$ lie in $|z| \geqslant k$, therefore, $Q(z)$ is a polynomial of degree $n$ having all its zeros in $|z| \leqslant k$. Applying Theorem 1 with $f(z)$ replaced by $P(z)$ and $F(z)$ by $Q(z)$, we obtain for every $R>r \geqslant k$ and $|z| \geqslant 1$,

$$
|B[P(R z)]+\psi(R, r, \alpha, \beta, k) B[P(r z)]| \leqslant|B[Q(R z)]+\psi(R, r, \alpha, \beta, k) B[Q(r z)]| .
$$

This proves Theorem 2 .

Proof of Theorem 3. Let $m=\min _{|z|=k}|P(z)|$. For $m=0$, there is nothing to prove. Assume that $m>0$, so that all the zeros of $P(z)$ lie in $|z|<k$ and we have,

$$
m\left|\frac{z}{k}\right|^{n} \leqslant|P(z)| \text { for }|z|=k .
$$


Applying Theorem 1 with $F(z)$ replaced by $P(z)$ and $f(z)$ by $m\left(\frac{z}{k}\right)^{n}$, we obtain for all real or complex numbers $\alpha, \beta$ with $|\alpha| \leqslant 1,|\beta| \leqslant 1, R>r \geqslant k$,

$$
\begin{aligned}
& |B[P(R z)]+\psi(R, r, \alpha, \beta, k) B[P(r z)]| \\
\geqslant & \frac{1}{k^{n}}\left|B\left[E_{n}(R z)\right]+\psi(R, r, \alpha, \beta, k) B\left[E_{n}(r z)\right]\right| \min _{|z|=k}|P(z)| .
\end{aligned}
$$

This proves Theorem 3.

Proof of Theorem 4. Let $M=\max _{|z|=k}|P(z)|$, then $|P(z)| \leqslant M$ for $|z| \leqslant k$. If $\lambda$ is any real or complex number with $|\lambda|>1$, then by Rouche's theorem the polynomial $G(z)=P(z)-\lambda M$ does not vanish in $|z|<k$. Consequently the polynomial

$$
H(z):=\left(\frac{z}{k}\right)^{n} \overline{G\left(\frac{k^{2}}{\bar{z}}\right)}
$$

has all zeros in $|z| \leqslant k$ and $|G(z)|=|H(z)|$ for $|z|=k$. On applying Theorem 1, we have for all real or complex numbers $\alpha, \beta$ with $|\alpha| \leqslant 1,|\beta| \leqslant 1, R>r \geqslant k, k \leqslant 1$ and $|z| \geqslant 1$,

$$
|B[G(R z)]+\psi(R, r, \alpha, \beta, k) B[G(r z)]| \leqslant|B[H(R z)]+\psi(R, r, \alpha, \beta, k) B[H(r z)]| .
$$

Since

$$
H(z):=\left(\frac{z}{k}\right)^{n} \overline{G\left(\frac{k^{2}}{\bar{z}}\right)}=\left(\frac{z}{k}\right)^{n} \overline{P\left(\frac{k^{2}}{\bar{z}}\right)}-\bar{\lambda}\left(\frac{z}{k}\right)^{n} M=Q(z)-\bar{\lambda}\left(\frac{z}{k}\right)^{n} M .
$$

Therefore, using the fact that $B$ is linear and $B[1]=\lambda_{0}$, we get from inequality (26)

$$
\begin{aligned}
& \left|B[P(R z)]+\psi(R, r, \alpha, \beta, k) B[P(r z)]-\lambda \lambda_{0} M(1+\psi(R, r, \alpha, \beta, k))\right| \\
\leqslant & \left|B[Q(R z)]+\psi(R, r, \alpha, \beta, k) B[Q(r z)]-\bar{\lambda} M \frac{1}{k^{n}}\left(B\left[E_{n}(R z)\right]+\psi(R, r, \alpha, \beta, k) B\left[E_{n}(r z)\right]\right)\right| .
\end{aligned}
$$

Using Corollary 2 for the polynomial $Q(z)$ and noting that $|P(z)|=|Q(z)|$ for $|z|=k$, we obtain

$$
|B[Q(R z)]+\psi(R, r, \alpha, \beta, k) B[Q(r z)]| \leqslant \frac{1}{k^{n}}\left|B\left[E_{n}(R z)\right]+\psi(R, r, \alpha, \beta, k) B\left[E_{n}(r z)\right]\right| M .
$$

Therefore, we can choose an argument of $\lambda$ in (27) such that

$$
\begin{aligned}
& \left|B[Q(R z)]+\psi(R, r, \alpha, \beta, k) B[Q(r z)]-\bar{\lambda} M \frac{1}{k^{n}}\left(B\left[E_{n}(R z)\right]+\psi(R, r, \alpha, \beta, k) B\left[E_{n}(r z)\right]\right)\right| \\
= & |\lambda| M \frac{1}{k^{n}}\left|B\left[E_{n}(R z)\right]+\psi(R, r, \alpha, \beta, k) B\left[E_{n}(r z)\right]\right|-|B[Q(R z)]+\psi(R, r, \alpha, \beta, k) B[Q(r z)]| .
\end{aligned}
$$


Using (28) in (27), we get

$$
\begin{aligned}
& \mid B[P(R z)]+ \psi(R, r, \alpha, \beta, k) B[P(r z)]|-| \lambda|| \lambda_{0}|M| 1+\psi(R, r, \alpha, \beta, k) \mid \\
& \leqslant|\lambda| M \frac{1}{k^{n}}\left|B\left[E_{n}(R z)\right]+\psi(R, r, \alpha, \beta, k) B\left[E_{n}(r z)\right]\right| \\
&-|B[Q(R z)]+\psi(R, r, \alpha, \beta, k) B[Q(r z)]| .
\end{aligned}
$$

Equivalently,

$$
\begin{aligned}
& |B[P(R z)]+\psi(R, r, \alpha, \beta, k) B[P(r z)]|+|B[Q(R z)]+\psi(R, r, \alpha, \beta, k) B[Q(r z)]| \\
\leqslant & |\lambda| M\left\{\left|\lambda_{0}\right||1+\psi(R, r, \alpha, \beta, k)|+\frac{1}{k^{n}}\left|B\left[E_{n}(R z)\right]+\psi(R, r, \alpha, \beta, k) B\left[E_{n}(r z)\right]\right|\right\} .
\end{aligned}
$$

Making $|\lambda| \rightarrow 1$, we have

$$
\begin{aligned}
& |B[P(R z)]+\psi(R, r, \alpha, \beta, k) B[P(r z)]|+|B[Q(R z)]+\psi(R, r, \alpha, \beta, k) B[Q(r z)]| \\
\leqslant & M\left\{\left|\lambda_{0}\right||1+\psi(R, r, \alpha, \beta, k)|+\frac{1}{k^{n}}\left|B\left[E_{n}(R z)\right]+\psi(R, r, \alpha, \beta, k) B\left[E_{n}(r z)\right]\right|\right\} .
\end{aligned}
$$

By the Maximum Modulus Principle for the polynomial $P(z)$ when $k \leqslant 1$, we get

$$
M=\max _{|z|=k}|P(z)| \leqslant \max _{|z|=1}|P(z)| \text {. }
$$

Combining (30) and (29), we get desired result.

Proof of Theorem 5. The desired result immediately follows by combining Theorem 2 and Theorem 4.

Proof of Theorem 6. If $P(z)$ has a zero on $|z|=k$, then the result follows from Theorem 5. Therefore we assume that $P(z)$ has all zeros in $|z|>k$, so that $m=$ $\min _{|z|=k}|P(z)|>0$ and for a real or complex number $\lambda$ with $|\lambda|<1$, we have $|\lambda m|<$ $m \leqslant|P(z)|$, for $|z|=k$. By Rouche's theorem, the polynomial $G(z)=P(z)-\lambda m$ does not vanish in $|z|<k$. Consequently the polynomial

$$
H(z):=\left(\frac{z}{k}\right)^{n} \overline{G\left(\frac{k^{2}}{\bar{z}}\right)}
$$

has all zeros in $|z| \leqslant k$ and $|G(z)|=|H(z)|$ for $|z|=k$. By applying Theorem 1, we have for all real or complex numbers $\alpha, \beta$ with $|\alpha| \leqslant 1,|\beta| \leqslant 1, R>r \geqslant k, k \leqslant 1$ and $|z| \geqslant 1$

$$
|B[G(R z)]+\psi(R, r, \alpha, \beta, k) B[G(r z)]| \leqslant|B[H(R z)]+\psi(R, r, \alpha, \beta, k) B[H(r z)]| .
$$


Substituting for $G(z)$ and $H(z)$ in (32), using the fact that $B$ is linear and $B[1]=\lambda_{0}$, we get

$$
\begin{aligned}
& \mid B[P(R z)]+ \psi(R, r, \alpha, \beta, k) B[P(r z)]-\lambda \lambda_{0} m(1+\psi(R, r, \alpha, \beta, k)) \mid \\
& \leqslant \mid B[Q(R z)]+\psi(R, r, \alpha, \beta, k) B[Q(r z)] \\
&-\bar{\lambda} m \frac{1}{k^{n}}\left(B\left[E_{n}(R z)\right]+\psi(R, r, \alpha, \beta, k) B\left[E_{n}(r z)\right]\right) \mid .
\end{aligned}
$$

Choosing the argument of $\lambda$ suitably, which is possible, we get from (33)

$$
\begin{gathered}
|B[P(R z)]+\psi(R, r, \alpha, \beta, k) B[P(r z)]|-|\lambda|\left|\lambda_{0}\right| m|1+\psi(R, r, \alpha, \beta, k)| \\
\leqslant|B[Q(R z)]+\psi(R, r, \alpha, \beta, k) B[Q(r z)]|-|\lambda| m \frac{1}{k^{n}} \mid B\left[E_{n}(R z)\right] \\
+\psi(R, r, \alpha, \beta, k) B\left[E_{n}(r z)\right] \mid
\end{gathered}
$$

This gives,

$$
\begin{aligned}
& |B[P(R z)]+\psi(R, r, \alpha, \beta, k) B[P(r z)]| \\
\leqslant & |B[Q(R z)]+\psi(R, r, \alpha, \beta, k) B[Q(r z)]| \\
& -|\lambda|\left\{\frac{1}{k^{n}}\left|B\left[E_{n}(R z)\right]+\psi(R, r, \alpha, \beta, k) B\left[E_{n}(r z)\right]\right|-\left|\lambda_{0}\right||1+\psi(R, r, \alpha, \beta, k)|\right\} m .
\end{aligned}
$$

Making $|\lambda| \rightarrow 1$, we have

$$
\begin{aligned}
& |B[P(R z)]+\psi(R, r, \alpha, \beta, k) B[P(r z)]| \\
\leqslant & |B[Q(R z)]+\psi(R, r, \alpha, \beta, k) B[Q(r z)]| \\
& -\left\{\frac{1}{k^{n}}\left|B\left[E_{n}(R z)\right]+\psi(R, r, \alpha, \beta, k) B\left[E_{n}(r z)\right]\right|-\left|\lambda_{0}\right||1+\psi(R, r, \alpha, \beta, k)|\right\} m,
\end{aligned}
$$

Also, by Theorem 4, we have

$$
\begin{aligned}
& |B[P(R z)]+\psi(R, r, \alpha, \beta, k) B[P(r z)]|+|B[Q(R z)]+\psi(R, r, \alpha, \beta, k) B[Q(r z)]| \\
\leqslant & \left\{\left|\lambda_{0}\right||1+\psi(R, r, \alpha, \beta, k)|+\frac{1}{k^{n}}\left|B\left[E_{n}(R z)\right]+\psi(R, r, \alpha, \beta, k) B\left[E_{n}(r z)\right]\right|\right\} \max _{|z|=1}|P(z)|,
\end{aligned}
$$

Combining the inequalities (35) and (36), we get the desired result.

Acknowledgements. We are grateful to the referee for his useful comments and valuable suggestions. 


\section{REFERENCES}

[1] N. C. Ankeny and T. J. Rivlin, On a theorem of S. Bernstein, Pacific J. Math., 5 (1955), 849-852.

[2] A. AzIZ, Inequalities for the polar derivatives of a polynomial, J. Approx. Theory, 55 (1988), 183193.

[3] A. AzIZ And Q. M. Dawood, Inequalities for a polynomial and its derivative, J. Approx. Theory, 53 (1988), 155-162.

[4] A. AZIZ AND W. M. SHAH, Some inequalities for the polar derivative of a polynomial, Indian Acad. Sci. (Math. Sci.), 107 (1997), 263-270.

[5] A. AzIZ AND B. A. ZARGAR, Inequalities for a polynomial and its derivative, Math. Inequl. Appl., 1 (4) (1998), 543-550.

[6] S. BERNSTEIn, Sur l'ordre de la meilleure approximation des fonctions continues par des polynomes de degré donnè, Mémoires de l'-Acadèmie Royale de Belgique, 4 (1912), 1-103.

[7] P. D. LAX, Proof of a conjecture of P. Erdôs on the derivative of a polynomial, Bull. Amer. Math. Soc. (N.S), 50 (1944), 509-513.

[8] M. Marden, Geometry of Polynomials, Math. Surveys, No. 3, Amer. Math. Soc., Providence, R.I. (1949).

[9] G. Pólya And G. Szegő, Problems and Theorems in Analysis, (New York: Springer-Verlag) (1972), Vol. 1.

[10] Q. I. Rahman, Functions of exponential type, Trans. Amer. Soc., 135 (1969), 295-309.

[11] Q. I. Rahman And G. Schmeisser, Les inégalités de Markoff et de Bernstein. Séminaire de Mathématiques Supérieures, No. 86 (Ete 1981). Presses de l’Université de Montréal, Montreal (1983), $173 \mathrm{pp}$.

[12] Q. I. Rahman and G. SchmeIsser, Analytic Theory of Polynomials, Oxford University Press, Oxford, 2002.

[13] M. RIESZ, Über einen Satz des Herrn Serge Bernstein, Acta Math., 40 (1916), 337-347.

[14] W. M. SнAн, A generalization of a theorem of Paul Turán, J. Ramanujan Math. Soc. 11 (1996), $67-72$.

[15] W. M. Shah And A. Liman, An operator preserving inequalities between polynomials, Journal of Inequalities in Pure and Applied Mathematics, 9 (1) (2008), Article 25, pp 8.

[16] W. M. SHAH AND A. LimAn, Integral estimates for the family of B-operators, Operators and Matrices, 5 (1) (2011), 79-87. 